\title{
Research on the Construction of Smart Pension System under the Background of Big Data
}

\author{
Le Gao ${ }^{1}$, Wanting Zhang ${ }^{1}$, Jia Li ${ }^{1}$, Tian Yang ${ }^{2 *}$ and Jianling Liang ${ }^{3}$ \\ ${ }^{1}$ Department of Intelligent Manufacturing, Wuyi University, China \\ ${ }^{2}$ Guangdong Qiaoxiang Culture Research Center, Wuyi University, China \\ ${ }^{3}$ School of Politics and Law, Wuyi University, China
}

*Corresponding author: Tian Yang, Guangdong Qiaoxiang Culture Research Center, Wuyi University, China.

\begin{abstract}
Based on the characteristics of China's large population base, the speed of China's aging population continues to grow, and the smart pension model has become the trend of China's pension service. This paper will analyze the existing problems of China's pension service from three pension models of home-based care, community care and institutional care, and analyze the development status and feasibility of smart pension service at home and abroad. On the basis of fieldwork, this study combined with the idea of big data to construct the structure of smart pension system from the infrastructure layer (IAAs), data and resource storage management layer (DAAS), basic service layer (PAAS) and business application layer (SaaS). The content of smart pension system includes smart medical system, smart community management system and other service systems. Finally, this paper puts forward some suggestions to realize the smart pension service system under the background of big data, which can provide reference for solving the problem of social pension.
\end{abstract}

Keywords: Big Data; Aging Population; Smart Pension

\section{Introduction}

China's economy is in a period of rapid development. On the one hand, the gradual acceleration of population mobility has led to many parents and children living in different places; on the other hand, most of the young people are too busy to take care of the elderly, so there are more and more Empty nest elderly (Generally refers to the children left home after the elderly) in China. In addition, in recent years, China's population aging rate continues to grow significantly (Figure 1). By 2019, the proportion aged 60 and above in China has accounted for $18.1 \%$ of the total population. By 2019, the proportion of the elderly aged 60 and above has accounted for $18.1 \%$ of China's total population. Compared with the historical data, the degree of population aging has increased by $0.2 \%$ annually from 2001 to 2010 and $0.4 \%$ annually from 2011 to
2018 [1]. In short, large base, fast growth, and poor overall health are the significant characteristics of China's aging population [2]. Based on the above two points, the problem of providing for the aged has become the focus of the Chinese society and the government in recent years, which will have a profound impact on the country's economic development, social stability and social security [3]. At present, China's pension model can be roughly divided into three types: home care, community care and institutional care model [4]. Chinese traditional culture and the old people's inherent idea of "returning to their roots" lead to $90 \%$ of the elderly will choose home-based care, while only $7 \%$ and $3 \%$ of the elderly choose community care and institutional care respectively [5]. Based on the above data, the key to solve China's pension problem is to solve 
the problem of $90 \%$ of the elderly who choose home-based care. Therefore, the promotion of smart pension model has become an urgent need of the society.

With the continuous development of information technology, the idea of "big data" promotes the progress of various fields of society [6]. For example, information means such as Internet and Internet of things have been proposed to be used in the field of elderly care; meanwhile, artificial intelligence means such as natural language processing, face recognition, machine vision and temperature sensing also bring new opportunities for China's pension service mode [7]. In China, the research on big data in smart pension can be divided into two categories [8]: first, to create a comprehensive intelligent home, such as emergency button sensor, panoramic language camera, intelligent household products, intelligent security, etc. the pension service mode of "system + service + elderly + terminal" can give full play to the role of big data platform and intelligent home [9]; second, the implementation of the "community + home" pension service mode, that is, the community relies on the "Internet plus" data platform, using community resources to provide personalized care services for the elderly in the community [10]. The construction and application of smart pension system under the background of big data is still in its infancy, and the effect of pension service still has a lot of room to improve. Therefore, this paper will build a smart pension system under the support of big data and provide help for China's smart pension problem.

\section{Problems in China's Pension Service}

\section{Problems in home care services}

The problems of home-based care services in China are mainly divided into the following three aspects: First, problems happen in daily life. The biggest problem for the elderly who choose to stay at home is the problem of daily life. For example, washing and cooking, indoor cleaning, daily travel, entertainment activities and so on. In order to reduce the financial burden of themselves and their children, most of the elderly people choose to take care of themselves, instead of hiring a nanny. Without the care of professionals, it is difficult for the elderly to achieve balanced diet, exercise, clean living environment and reasonable allocation of their entertainment time. Especially with the continuous improvement of social living standards, the elderly eats a lot of high oil, high sugar food for a long time, and develop the habit of playing mobile phones, computers, watching TV and so on. In the long run, bad diet and living habits will lead to the risk of chronic diseases among the elderly, such as diabetes, cardiovascular and cerebrovascular diseases and even cancer.

Second, problems happen on medical care. It is the most common psychological demand of the elderly to have a doctor conveniently. The medical treatment and recuperation of the elderly at home is the core problem for the aged people who stay at home. For home-based elderly care, people can be divided into two categories: those who with partners and without partners. For the former, if emergencies happen, such as heart disease, sudden falls and other situations, their partners will help to dial emergency call in time, but this will also affect the psychology healthy of his or her partner, too. For the latter, however, if this kind of elderly people in the face of emergencies, the situation will be extremely dangerous. The elderly is likely to miss the best treatment time. However, if they choose to recuperate at home during the period of rehabilitation, they will face the problems of lacking professional care and rehabilitation plan, which will also affect the subsequent physical recovery.

Thirdly, problems happen on spiritual consolation. The topic of caring for the empty nest elderly has always been a hot topic in China. Some people think that the elderly choose home-based care is because their children do not fulfill their obligation to support the elderly, which leads to the intensification of homesociety contradictions. For the elderly, what they want more is the company of their children. However, the younger generation are busy with their work and family under the pressure of life, so that they don't have enough time and energy to care of their parents, so the psychological and spiritual needs of the elderly at home will increasing. In this case, the loneliness happens in mental life of the elderly at home will directly affect their health, and hence indirectly affect their physical healthy.

\section{Problems of community endowment service}

The problems faced by the community pension service in China are mainly divided into the following three aspects: First, the combination of medical and nursing is not enough. With the decline of the physical condition and physical fitness of the elderly, the demand for medical treatment and daily life health care of the elderly is also increasing. The community pension service can only provide some periodic arrangements for the elderly inspection, safety and health popularization and other services which is far more not enough. Because the community pension service lack of the combination of medical care and pension, they also don't have basic medical equipment, and no professional community medical personnel are responsible for the daily medical treatment, no psychological consultation, emergency medical treatment and other services for the elderly. Therefore, it leads to the serious shortage of the combination of medical care and pension in the current community pension service, which leads to the problem of medical care and pension for the elderly cannot be well solved.

Second, insufficient participation of social forces. The mechanism of social forces participating in community pension service still needs to be improved. The government does not have a sound set of laws and regulations to supervise the community 
pension service, which leads to the loose and irresponsible community pension service. Moreover, there is not much interaction and concern between the community volunteers and community staff and the daily life of the elderly, and a large part of social forces are not involved in the community pension service, so the efficiency and quality of the community pension service are not high, and it does not reach our ideal state [11].

Third, Supply and demand dislocation of pension service. The focus of community pension service is often on the daily diet, spiritual life and cultural activities of the elderly. The service for each elderly is convergent, and there is no personalized service for the elderly. For example, some elderly people have a greater demand for on-site medical services, and some others have a greater demand for spiritual life, but the community pension service is still in a dilemma. The unified service cannot meet the special needs of every elderly, so it will lead to the problem of supply and demand dislocation of community pension service.

\section{Problems of institutional care for the aged}

The existing problems of China's pension service are mainly divided into the following three aspects: First, the cost of providing for the aged is high. Institutional care mainly refers to those who choose to go to nursing homes. In China, only 3\% of the elderly is likely to choose institutional care, the main reason is that institutional care needs the elderly to bear a pension cost. The present situation of the elderly in China is "getting old before getting rich". There are also a large number of elderly people who have no pension and other income. They can only consume their own savings or rely on their children's alimony when they enter the elderly life, so the cost of institutional pension is not a small expenditure for the elderly. It is for this reason that most of the elderly cannot afford the institutional care.

Second, problems happen on living environment. At present, one of the main problems of institutional care for the aged is the living environment and health problems in nursing homes. The service mode of the nursing home belongs to the service oriented to the living groups. There will be many elderly people who are difficult to take care of themselves in the nursing home. Although the health and environment of the nursing home will be in the charge of the cleaning staff, most of them have not received professional training, which leads to the health of the nursing home is difficult to guarantee. The elderly living in the environment of poor health conditions for a long time will affect their physical and mental health.

Third, Problem happens on spiritual consolation of the elderly. Due to the influence of traditional Chinese culture, children are considered to have obligation to support and take care of their elderly parents. However, most of the contemporary young people are busy with their own work and family and have no time and energy to take care of their parents. A small number of people will send their parents to nursing homes. Without the company of children, the psychology healthy of the elderly will be affected by the loneliness environment. Therefore, the spiritual comfort of the elderly in nursing homes is also a big problem.

\section{Development Status and Feasibility Analysis of Smart Pension}

\section{Development status of smart pension}

Smart pension refers to the use of Internet, Internet of things, big data, cloud computing and other technologies to develop an intelligent service system platform for the elderly. This platform provides convenient, efficient and personalized services for the elderly, and can form a targeted and sustainable smart pension business model according to different pension methods and needs. With the help of different social forces, such as hospitals, governments, communities and institutions, the system will improve the service quality and business level of the elderly care service team, making the system construction of home-based care, community-based care and institutional care more scientific. While bringing technology enjoyment to the elderly, it can also improve the quality of life of the elderly and ensure their later life.

At present, China's smart pension model is in its infancy. In the exploration of smart pension mode supported by big data, researchers integrate big data technology into smart pension platform, analyze the needs of pension cloud platform, and construct a platform framework of perception layer, application layer, interface layer, business processing layer and data layer to explain the construction and operation of the platform [12]; Zhou analyzed the definition, significance and feasibility of cloud service platform for home-based elderly care for the elderly under the background of big data [13]; some scholars discussed the combination mode of community elderly care and medical care based on big data technology, and divided the mode scheme into three parts: data collection and processing, data analysis and visualization, and data feedback and re collection [14].Xu and Fang conceived the intelligent home care system under the situation of "Internet plus" and put forward the method of building an intelligent pension system. He also proposed that personnel training and pilot projects should be carried out to achieve intelligent retirement [15]. Jia proposed that under the background of smart city construction, to build a new big data-driven pension model relying on big data and end network cloud technology is a choice in the digital era [16]. The development of western developed countries in the field of smart pension has been on the right track, and the types of smart pension products are relatively perfect. In the UK, it mainly integrates the prototype of health and social health care services, relies on the community to establish smart care centers, and realizes smart care services for the elderly through the Internet and wearable devices [17]. The U.S. government introduced pension services into the market 
competition mechanism, and encouraged Apple, Samsung and other technology companies to actively develop pension products. Due to the development of the Internet and the fierce competition of enterprises, all kinds of smart pension products in the United States have gradually become perfect, and the development process is also accelerating [18]. Japan, as a country with the most aging population, has already realized the scientific and technological pension. And it has a relatively sound legal system in terms of pension, which has laid a strong foundation for the development of smart pension [19]. Meanwhile, Germany creates an intelligent technology platform to realize home-based care and institutional care and improves the living environment of the elderly by using a variety of intelligent devices to assist them in their life [20].

\section{Feasibility analysis of smart pension}

China is a country that entered the aging society later, but according to the characteristics of the population structure, it can be predicted that China will be in the aging stage for a long period of history. With the rapid growth of population aging and the emerging problems of contemporary pension service, China is forced to construct a new pension service model with Chinese characteristics. In recent years, China has always been in a positive attitude in solving the problem of providing for the aged. Solving the problem of providing for the aged is to solve the problem of people's livelihood. The intelligent pension system has the characteristics of hierarchy, structure and informational, which meets the needs of pension services.

\section{Construction of Smart Pension System under the Background of Big Data}

\section{The overall structure of smart pension system under the} background of big data

The structure of the current smart pension system shows a variety of forms due to different regions, infrastructure and user needs. Each system exists independently, and the same architecture cannot get the same good effect in different environments.
Although there are some differences between each system, the overall architecture, design concept and main technology are similar. The whole information platform of smart pension system cannot be built without the foundation of big data. According to the structure level of big data architecture, it can be divided into infrastructure layer (IAAs), data and resource storage management layer (DAAS), basic service layer (PAAS) and business application layer (SaaS). Therefore, the overall structure of smart pension system in the context of big data is shown in Figure 2. From the content of big data smart pension system, it is mainly divided into three parts: smart medical system, smart community management system and other service systems. The framework of smart pension system under the background of big data, which is composed of the supply side, the demand side and the intelligent pension service platform supported by big data, is shown in Figure 3. The smart elderly care service platform supported by big data is responsible for the demand analysis of the service demand of the elderly, and the supply and demand matching and resource scheduling for the demander and the supplier (Figure 3).

Infrastructure-as-a-Service (IaaS): The infrastructure layer is the bottom layer of the big data architecture, which is the basis for realizing all functions. It includes server, storage, network and other facilities. These resources are pooled through virtualization technology to provide specific services, computing and other functions for other layers. The infrastructure layer architecture is shown in Figure 4.

Data-as-a-Service (DaaS): The data and resource storage management layer mainly stores all the data in the smart pension system, such as smart medical data, health management data, personal file data of the elderly, catering data, smart home data, etc. The database should realize the sharing principle. For example, hospitals, communities and major institutions should be connected to each other, so as to grasp the information of the elderly in time and realize the real-time protection of the elderly. The structure of data and resource storage management layer is shown in Figure 5.

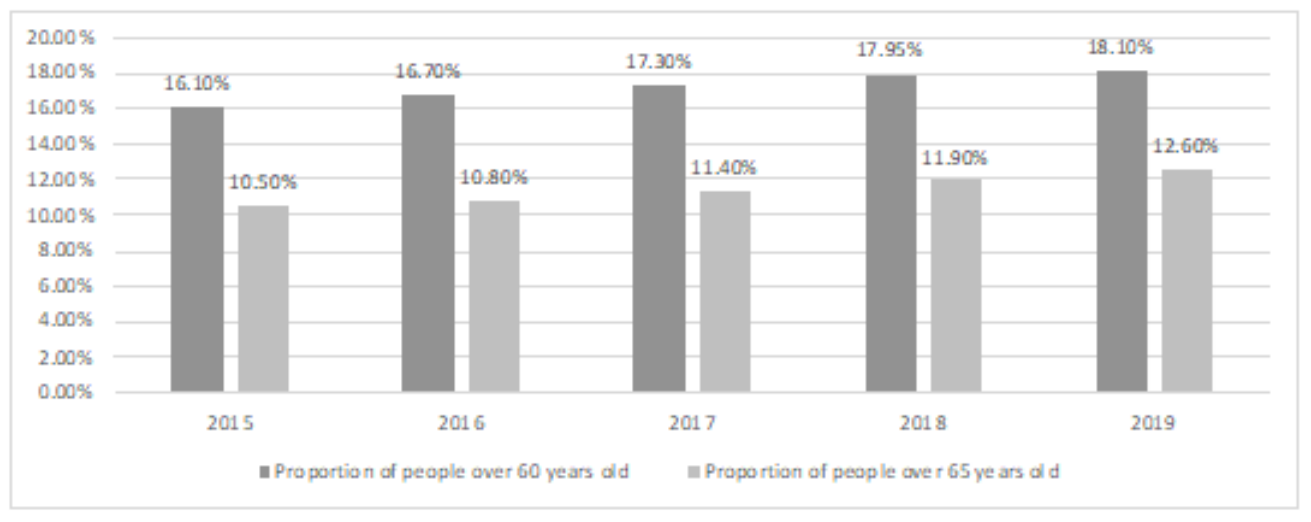

Figure 1: Trend of China's Population Aging Data in Recent Five Years (Data from the National Bureau of Statistics). 


\section{Smart pension service platform supported by big data}

\section{Software-as-a-Service (SaaS)}

\section{Platform-as-a-Service (PaaS)}

Data-as-a-Service (DaaS)

Infrastructure-as-a-Service (IaaS)

Figure 2: Overall Structure of Smart Pension System under the Background of Big Data.

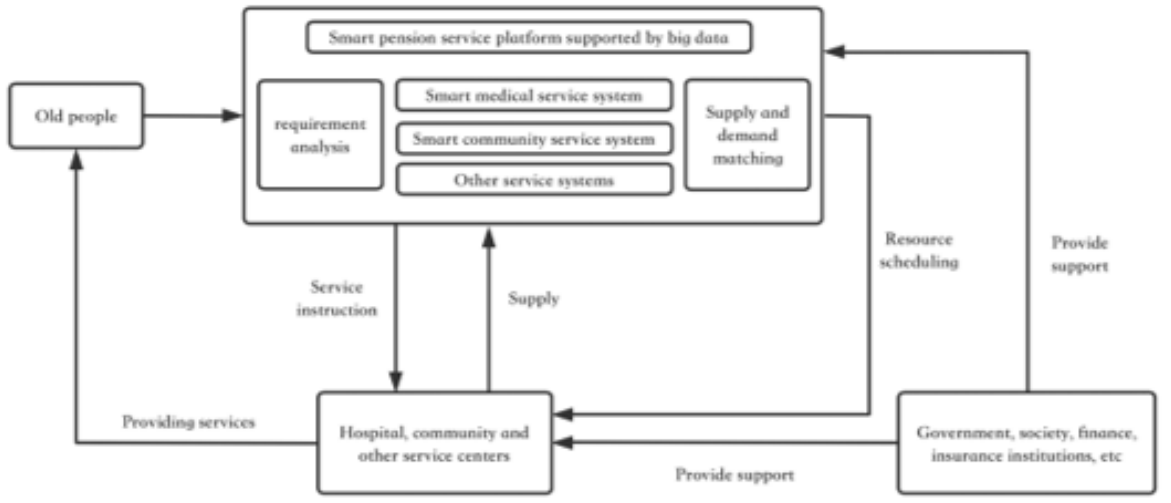

Figure 3: Framework of Smart Pension System under the Background of Big Data.

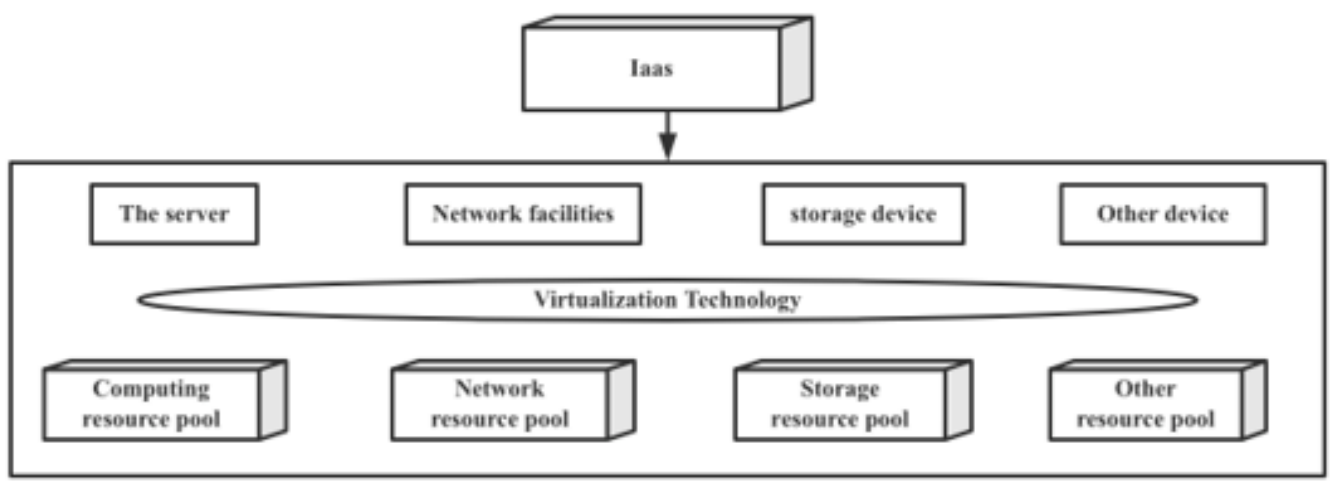

Figure 4: Framework of the Infrastructure Layer. 


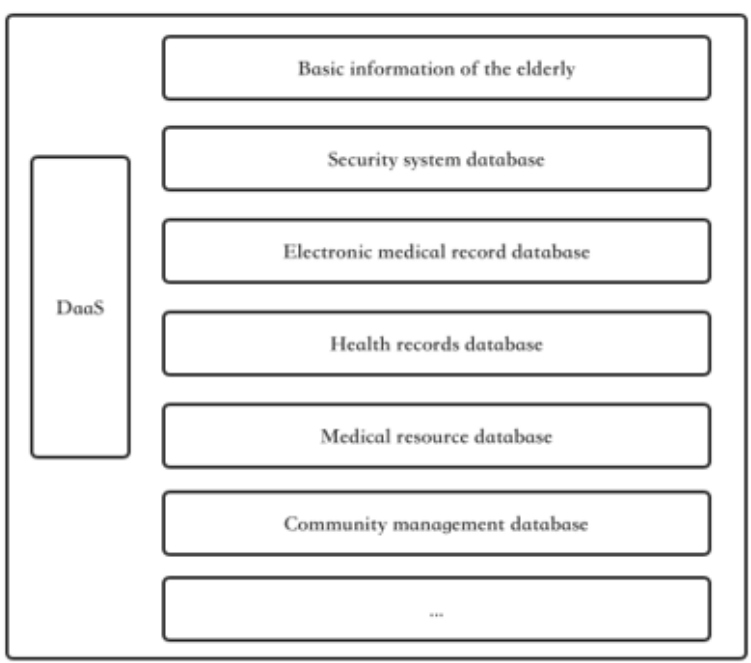

Figure 5: Architecture of Data and Resource Storage Management Layer.

Platform-as-a- Service (PaaS): From the perspective of application deployment of basic service layer, under the premise of the content of smart pension system under the background of big data, the basic service layer will be divided into smart medical service platform, smart community management platform and other service platforms. Each platform will realize information sharing, unified authority, application development and other functions. The architecture of the basic service layer is shown in
Figure 6.

Software-as-a- Service (SaaS): The business application layer is mainly oriented to the elderly users to achieve the needs of the elderly users, and provide all-round services for the elderly through the Internet and the combination of software and hardware, such as emergency rescue, family remote video and voice, medication reminder, wearable devices, etc. the architecture of the business application layer is shown in Figure 7.

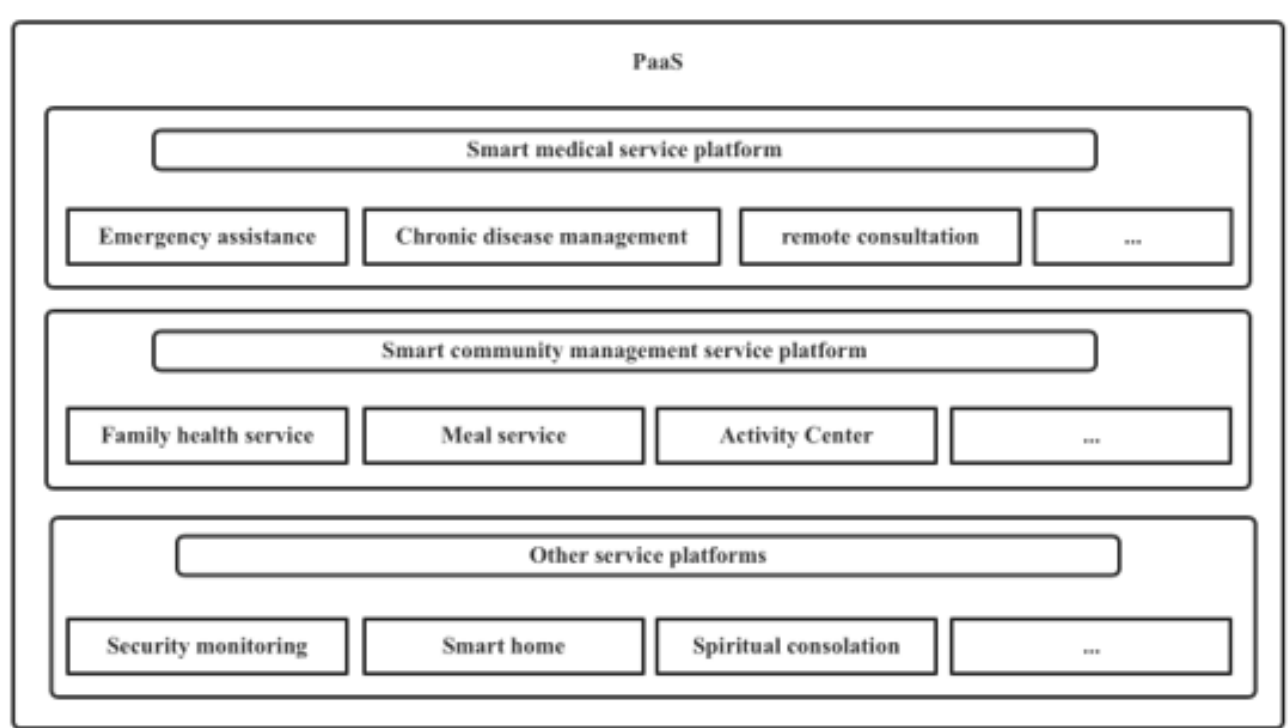

Figure 6: Basic Service Layer Architecture.

\section{Smart medical system and smart community under the} background of big data

Intelligent medical system mainly includes intelligent hospital, long-range medical treatment, emergency rescue, health management and other functions. The focus of smart pension mainly lies in the smart medical system. For example, the emergency rescue function of smart medical system will be realized through various wearable devices and sensor devices of the elderly. Emergency help equipment for the elderly can obtain real-time information of the elderly from big data platform, such as blood 
pressure, blood glucose, vital signs and so on. In case of emergency happens, immediately alert will connected to the hospital management system to take necessary measures; the elderly can also conduct online consultation, describe their condition through connecting with the doctor of the hospital, and the doctor can provide the whole process services such as consultation, prescription, offline delivery and reminding to take medicine for the elderly according to the health information data and past medical history data of the elderly. Under the promotion of smart city, smart health applies big data, cloud computing, Internet of things, data computing and other technologies to smart health system. Although the current theoretical system of smart medical is not perfect, smart medical is an important prerequisite for the realization of smart pension, the key to its development lies in the support of technology and industry.

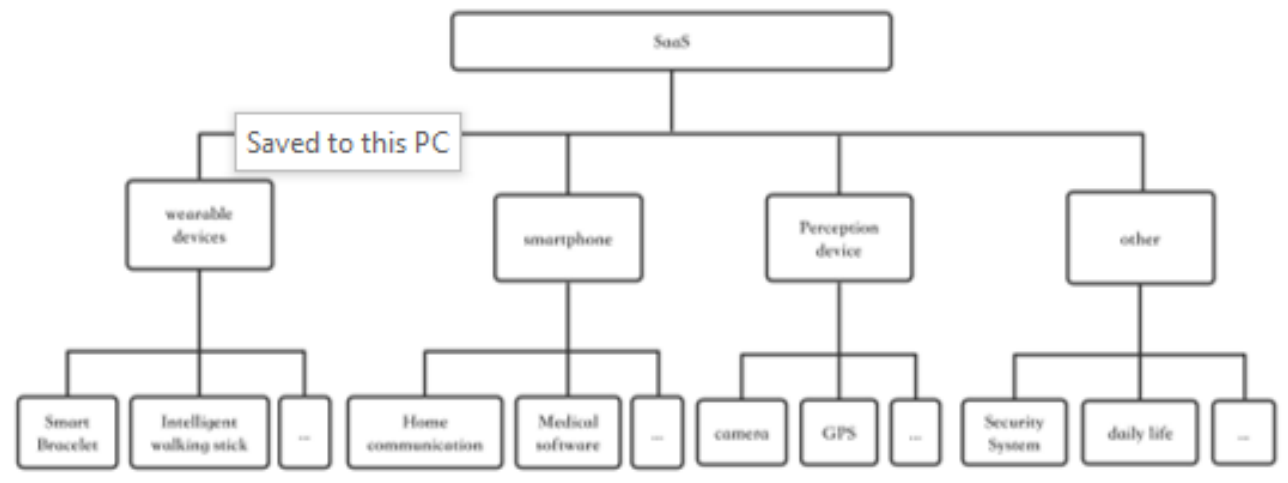

Figure 7: Business Application Layer Architecture.

Smart pension system should give full play to the role of the community. For example, the elderly family security system, regular physical examination prompt, etc. can use the strength of the community for block management and monitoring. Smart community management system can play the role of an intermediary in the smart pension, which can manage the health data of the elderly with the elderly in a community as a whole. And through the interconnection with nearby hospitals, meal delivery institutions and health service institutions, and through the linkage of various communities and nearby resource data, the big data smart pension platform of the whole city is jointly formed. In order to schedule resources more efficiently, several communities can be divided into one platform for management. In addition to hospitals and communities can play a greater role in the smart pension system, there are many institutions and products essential to the realization of smart pension. For example, smart home system, spiritual comfort platform, etc. In China, the production of smart home products comes out in an unending flow. Smart home for the elderly, such as intelligent security, intelligent lighting, voice control products, etc., will provide support for smart medical and smart community; spiritual comfort platform is for the elderly, such as online chat platform, family video, elderly university, etc., to make the elderly life no longer lonely and boring.

\section{Suggestions on Smart Pension under the Background of Big Data}

In order to realize the sustainable development of China's pension industry under the background of big data and improve the quality of pension services, it should be based on information construction, guided by government decision support, driven by the innovation of smart pension products, and supported by the training of professional service personnel. Make full use of social resources, build a smart pension service platform under the background of big data, and let the elderly live an intelligent and information-based old age life. The suggestions are mainly divided into the following four aspects:

First, Construction of information platform. As China's smart pension industry has just started, the construction of smart pension system is not perfect, the infrastructure is not perfect, and the traditional pension mode is limited by region and time, the quality of pension service in China is not optimistic. With the rapid development of big data and cloud computing technology, the realization of smart pension must be based on the information construction, such as positioning system, security system, medical system, etc. all need information management, and the data collection and processing of each system need to be based on the information construction. At the same time of information construction, we should strengthen the protection of information for the elderly; enhance the awareness of network security, in order to prevent data leakage.

Second, Government decision support. At present, the laws and regulations of China's pension service industry are not perfect. In order to comprehensively deal with the problem of population aging, our government should vigorously promote the development of pension service industry, take the government as the main force, 
and encourage social forces to participate in the pension service industry; We should improve the legal system of pension service industry, adhere to the principle of people-oriented, ensure the healthy development of pension service industry, and protect the legitimate rights and interests of the elderly; the government should provide financial support for pension service industry, and reasonably allocate all aspects of social resources; the government should establish the pilot of smart pension service, and promote the development of smart pension service.

Third, Promote the innovation of smart pension products. China's development speed in the field of smart home and smart electronic products is accelerating. With the arrival of $5 \mathrm{G}$ era, the development prospect of smart products will be more considerable. Various kinds of wearable devices, smart sensing devices and other smart products will enter every corner of our lives. For example, the now popular smart bracelet can detect the blood pressure and blood glucose of the elderly, and feedback the detection data to the hospital, so that the hospital can monitor the physical condition of the elderly; the geographic location of the elderly can be monitored through the GPS navigation system. Therefore, the innovation of intelligent products will provide a strong driving force for the construction of intelligent elderly care service platform.

Fourth, Professional training. The development of smart pension industry needs a large number of professional technical talents, including data mining, software development, big data operation and other professional talents, so we must attract social talents and encourage them to participate in the pension service industry. At the present stage, the number of people serving the pension industry is small and the quality is low. The biggest difference between smart pension and traditional pension is the quality of service. In order to ensure the quality of pension service, it is necessary to carry out professional training for service personnel, such as community managers, hospital nurses and so on. Improve the comprehensive quality of service personnel, formulate corresponding policies, improve the salary and welfare of service personnel, and attract more talents to join the team of smart pension service industry.

\section{Acknowledgement}

This project is supported by “Wuyi University Hong Kong Macao Joint Research and Development Fund: 2019WGALH23" and "Wuyi University Youth Research Group Fund:2019td10".

\section{Conflict of Interest}

No conflict of interest.

\section{References}

1. Ren Zeping, Xiong Chai, Zhou Zhe (2020) Characteristics and Trends of Population Aging in China. China Gerontology Daily, (004).

2. Zhou Rong, Zhuang Rulong, Huang Chenxi (2019) Evolution and Formation Mechanism of Population Aging Pattern in China. Acta Geographica Sinica 74(10): 2163-2177.
3. Ren Chong (2014) Impact of Aging on China's Social and Economic Development and Countermeasures. Inner Mongolia Social Sciences (Chinese Version) 35(5): 160-166.

4. Jiang Ling (2006) The choice of Chinese urban pension mode. Economic Forum 11: 51-53.

5. Jia Ruifang (2020) Construction of Smart Home Care Model of "Combination of Medical Care and Nursing Care" in Jiangsu Province under the Background of Big Data. General Nursing 18(9): 1120-1122.

6. Zhu Xiao-ning,Song Qiao (2017) An Analysis of Grid Construction Model of Smart Pension Service in China[A]. University of Ghana (UG), Ghana Institute of Management and Public Administration (GIMPA), University of Cape Coast (UCC), American Society for Public Administration (ASPA), University of Electronic Science and Technology of China (UESTC). Proceedings of 2017 International Conference on Public Administration(12th) \& International Symposium on West African Studies (1st) (Volume II) [C], 2017:6.

7. Sui Dangchen, Liu Xingchen (2020) Construction of Artificial Intelligence Home Care Model. Chongqing Social Sciences 7: 6-19+2.

8. Wang Sujuan, Li Hui, Wang Shujun, Yao Jingyi (2020) Research on the Construction of Cloud Service Platform for Home Care under the Background of Big Data. Cooperative economy and technology 13: 188190.

9. Zhang Yi, Xin Huan (2016) Research on Home Care Model based on Smart Home. Journal of Lanzhou Institute of technology 23(5): 36-39.

10. Liu Mengyun, Hu Yangfen (2020) Research on Home Care Model in Smart Community. Modern Communication 11: 72-74.

11.Wang Xin, Wang mingshou (2020) Collaborative Construction of Community Elderly Care Service System under the Background of Big Data. Journal of Lanzhou University (Social Science Edition) 48(1): 3645.

12. Zhai Yue, Zeng Weijia (2020) Design of Smart Pension Cloud Platform Based on Big Data Technology. Science and Technology Innovation 10: 55-56.

13. Zhou Na (2020) Construction Strategy of Cloud Service Platform for Elderly Home Care based on Big Data. Computer knowledge and technology 16(17): 92-94.

14. Jia muxiao, Yang Fan, Wang Jingjing (2020) Exploring the Mode of Community Elderly Care Combined with Medical Care based on Big Data Technology. Smart city 6(7): 12-14.

15. Home furnishing, Xu Kai, Fang Wen Jie (2019) Conception of the Intelligent Home Care Mode under the Internet Plus Situation. Science and Technology Innovation Guide 16(25): 240-242.

16. Jia Yan, LAN Zhiyong, Liu Runze (2020) Precision Pension: A New Pension Model Driven by Big Data. Journal of Public Management 17(2): $95-103+171$.

17. Morciano M, Checkland K, Billings J, et al. (2020) New integrated care models in England associated with small reduction in hospital admissions in longer-term: A difference-in-differences analysis [J] Health Policy 124(8)

18. Zhao Ning, Zhang Jian (2020) Experience and Enlightenment of Foreign Smart Pension Development Model. Social Science Trends 8: 67-71.

19. Lee SH, Ogawa N, Matsukura R (2016) Japan's pension reform, labor market responses, and savings[J]. Journal of the Economics of Ageing, pp. 67-75.

20. Robert Fenge, Francois Peglow (2018) Decomposition of demographic effects on the german pension system. The Journal of the Economics of Ageing 12: 61-76. 